\title{
UN HITO EN EL COLECCIONISMO INSTITUCIONAL CONTEMPORÁNEO EN ANDALUCÍA: LA FORMACIÓN DE LA COLECCIÓN DE ARTE DE LA FUNDACIÓN CAJASOL
}

\author{
A MILESTONE IN CONTEMPORARY INSTITUTIONAL \\ COLLECTING IN ANDALUSIA: THE FORMATION OF \\ THE CAJASOL FOUNDATION ART COLLECTION
}

\author{
Juan María Vélez Alvez \\ Universidad de Sevilla. España \\ ORCID: 0000-0003-4590-9683 \\ jvelez@us.es
}

Los orígenes de la Colección Cajasol se remontan a la fundación en 1842 de la primera de las cajas de ahorros que conformarían la entidad. Durante su historia se crea un conjunto de obras fruto de diferentes criterios de adquisición. Se trata de un proceso singular en el coleccionismo puesto que confronta diversos puntos de vista y criterios de adquisición que tienen como resultado una misma colección. Los fondos superan las cuatro mil obras, piezas que ofrecen una visión amplia del arte andaluz principalmente, aunque con unos planteamientos geográficos que disemina las fronteras cuando aborda lo contemporáneo. La cronología marca un amplio recorrido, desde el siglo XVII, con maestros del arte Barroco, al siglo XXI, con los nuevos lenguajes plásticos.

Palabras clave: coleccionismo; coleccionismo andaluz; coleccionismo institucional; Cajasol; gestión del patrimonio.

The origins of the Cajasol Collection date back to the foundation in 1842 of the first of the savings banks that would make up the entity. During its history a set of works is born fruit of different acquisition criteria. It is a unique process in collecting, as it confronts different points of view and acquisition criteria, which result in the same collection. The collection funds exceed four thousand works, pieces that offer a broad view vision of Andalusian art mainly, although with geographical approaches that disseminate the borders when addressing the contemporary. The chronology marks a long journey from the $17^{\text {th }}$ century, with masters of Baroque art, to the $21^{\text {st }}$ century, with the new plastic languages.

Keywords: collecting; Andalusian collecting; institutional collecting; Cajasol; heritage management. 


\section{HISTORIA DE LA ENTIDAD Y SUS FONDOS ARTÍSTICOS}

La Caja de Ahorros Cajasol nace el 18 de mayo de 2007. Su denominación oficial es Monte de Piedad y Caja de Ahorros San Fernando de Huelva, Jerez y Sevilla. Se trata de una entidad, al igual que todas las cajas de ahorros, privada, de crédito, de naturaleza fundacional y de carácter social, sin ánimo de lucro, que orienta su actividad a la consecución de fines de interés público. Resulta de la fusión entre el Monte de Piedad y Caja de Ahorros de Huelva y Sevilla, cuyo nombre comercial sería El Monte, y la Caja de Ahorros Provincial San Fernando de Sevilla y Jerez, conocida como Caja San Fernando, ambas radicadas desde sus orígenes en Andalucía. Las dos entidades son también el resultado de diversas integraciones a lo largo de la historia, lo que hace que Cajasol lleve ejerciendo su actividad financiera y social de manera ininterrumpida desde el año 1842, año de fundación del Monte de Piedad de Sevilla ${ }^{1}$, hasta 2011, fecha en la que su negocio financiero es absorbido por Caixabank.

El legado artístico generado por la caja andaluza pasa a engrosar el patrimonio de la actual Fundación Cajasol, entidad constituida en 2014 como fundación de carácter ordinario. Parte de la actividad de la fundación se dedica al mantenimiento del patrimonio cultural y la obra social que anteriormente desarrollaba la caja. En la actualidad, es una fundación privada denominada Fundación Monte de Piedad y Caja de Ahorros San Fernando de Huelva, Jerez y Sevilla, y cuyo nombre comercial es Fundación Cajasol.

A lo largo de este dilatado recorrido histórico, las instituciones que conformaron Cajasol han ido acumulando multitud de obras para su colección de arte, hoy renombrada como Colección de la Fundación Cajasol, que cuenta con 4.351 piezas $^{2}$. Se trata de la mayor colección privada de arte en Andalucía.

Estas obras no tuvieron en sus orígenes una vocación de colección de arte, se trataba de una mera acumulación de piezas artísticas sin un objetivo museológico definido ni criterios de adquisición o gestión profesionalizados. Los nuevos fondos llegaban a la institución respondiendo a motivos dispares: retratos conmemorativos de los fundadores de la institución ${ }^{3}$, adquisiciones destinadas a decoración, obras que se quedaban en propiedad del Monte de Piedad por depósitos de empeños que no eran retirados o embargos por operaciones de activo

${ }^{1}$ Campayo Rodríguez, 1992: 12.

2 Cifra que corresponde con el resultado final del inventario dirigido por Juan María Vélez Alvez, finalizado en el mes de mayo de 2014, y las cinco obras adquiridas desde ese año al momento actual (octubre de 2019).

${ }^{3}$ Por parte del Monte de Piedad de Sevilla, se conservan los retratos de Don Francisco Moreno Zaldarriaga y Don Domingo Pérez de Ansoátegui, de los pintores románticos José Gutiérrez de la Vega y Manuel Wssel respectivamente. Caja San Fernando aporta los realizados por el pintor sevillano Luis Sevil a Don Rafael Rivero de la Tegera y Don Cayetano Fernández González. 
impagadas. La aparición de las obras sociales de las cajas de ahorros a partir de $1970^{4}$ amplió los sistemas de adquisición mediante la organización de certámenes de artes plásticas cuyas obras premiadas eran adquiridas 5 . Además se propiciaron políticas expositivas en las que se cedían las salas de exposiciones a artistas a cambio de la donación de una obra para sus fondos.

Hasta mediados de la década de los noventa del siglo XX, las cajas de ahorros no toman conciencia del patrimonio acumulado. La primera actuación es encargar la elaboración de un inventario para conocer la magnitud cuantitativa de los fondos. En 1993, El Monte encarga a Francisco Pérez Valencia ${ }^{6}$ la elaboración del primer inventario de patrimonio artístico. Las obras consideradas como las más emblemáticas se publican en el catálogo titulado Colección El Monte ${ }^{7}$, que muestra parte del inventario realizado y que, por primera vez, utiliza de manera oficial la nomenclatura de colección de arte para definir los fondos de la caja de ahorros.

A finales de la misma década, concretamente en el año 2000, hace lo propio la Caja San Fernando, encargando la citada labor a Francisco del Río ${ }^{8}$.

Los inventarios resultantes, más allá de suponer un listado de obras acumuladas sin criterio específico alguno, acentúan el carácter de oficialidad de la colección, la consolidan a nivel institucional y actúan como punto de partida para su posterior desarrollo. Las dos entidades ponen en marcha mecanismos para profesionalizar la gestión y adquisición de fondos, cada una con métodos diferentes pero ambas con un marcado perfil profesional.

\section{LOS NOMBRES PROPIOS DEL PROYECTO: FRANCISCO PÉREZ VALENCIA Y FRANCISCO DEL RÍO}

La profesionalización de las estructuras destinadas a la gestión de las colecciones de arte tiene como hito clave el nombramiento de dos profesiones de perfil técnico como responsables del proyecto tanto en El Monte como en la Caja San Fernando.

En el caso de El Monte, Francisco Pérez Valencia ejerce durante catorce años como conservador-director de la Colección de Arte, cargo que se prolonga tres años más con la marca Cajasol. Doctor en Bellas Artes por la Universidad de Sevilla, cuenta con una prolífica carrera profesional relacionada con las artes plásticas. Como gestor planifica la política de acrecentamiento y difusión de la Colección Cajasol. Su papel en la adquisición de nuevas obras configura el diseño

${ }^{4}$ Palomo Pachón, 2004: 235-236.

5 Martín Martín, 2010: 479-489.

${ }^{6}$ Doctor en Bellas Artes, con 24 años de edad comienza su vinculación con la colección de arte El Monte.

${ }^{7}$ Pérez Valencia, 1994.

${ }^{8}$ Díaz Urmeneta/Marina/Ramos, 2012: 15. 
actual de los fondos. Fue responsable de la creación y dirección del Espacio ESCALA, Centro de Arte y sede de la Colección Cajasol (2007-2010). Ha publicado numerosos ensayos y textos sobre museografía. Sus libros La insurrección expositiva (Gijón, 2007) y Tener un buen Plan (Gijón, 2010), un manual crítico sobre los planes museológicos de las colecciones, son referencia específica en su disciplina. En la actualidad compagina su labor creativa como artista plástico con la docente, siendo profesor en la Universidad Loyola de Andalucía.

En la Caja San Fernando el inicio de unos criterios profesionales en la gestión y crecimiento de su colección de arte también se vincula a la labor de un profesional, en este caso Francisco del Río (Sevilla, 1955-2011). Licenciado en Historia del Arte, su trabajo siempre estuvo ligado al mundo de la cultura y, más concretamente, al de las artes plásticas. Colabora, a partir de 1973, con la galería de Juana de Aizpuru, para pasar con posterioridad a realizar prácticas en el Museo de Arte Contemporáneo. En el año 1981 es contratado para trabajar en la Delegación de Cultura del Ayuntamiento de Sevilla, donde coordina diferentes actividades culturales hasta el año 1983. Fue uno de los iniciadores del suplemento Culturas que acompañó los primeros pasos del Diario de Sevilla. Definitivamente recalará en el que fue su destino laboral definitivo, la Obra Social y Cultural de la Caja San Fernando.

En sus más de diez años al frente del Departamento de Artes Plásticas de la Obra Social de Caja San Fernando desarrolló una programación basada en cuatro pilares fundamentales, como son la atención a los artistas andaluces, puesta en marcha del Certamen de Artes Plásticas, exposiciones de divulgación de temas o autores de relevancia y proyectos de experimentación. Entre estos últimos fue destacable la labor de relacionar el flamenco con el arte contemporáneo mediante la fotografía y el vídeo.

\section{EL NACIMIENTO EFECTIVO DE LAS COLECCIONES: IMPLANTACIÓN DE CRITERIOS DE ADQUISICIÓN}

En el año 2000, una vez concluido el inventario en Caja San Fernando, Francisco del Río propone iniciar el proyecto de colección. Este lo contextualiza de manera muy precisa, delimita tanto el ámbito geográfico, en este caso Andalucía, como la cronología, las manifestaciones artísticas de la segunda mitad del siglo XX.

En cuanto al concepto de colección, pasó de las piezas aisladas a la adquisición de grupos amplios de obras de diversos autores. Estas pequeñas colecciones de cada artista debían recoger la trayectoria de cada uno de ellos, desde los pasos iniciales a las piezas más recientes, desarrollando una visión global de su lenguaje plástico. 
Se crea una comisión de compras, dependiente de la Obra Social y Cultural, para decidir sobre los autores y las obras a adquirir ${ }^{9}$. El inicio de un proyecto de compras supone la primera idea de colección por parte de esta entidad. El nacimiento oficial de la misma se produce con el primer grupo de obras adquiridas de la pintora Teresa Duclós. El texto institucional del catálogo que sirve para presentar la compra evidencia esta idea: "Es para mí una satisfacción presentar esta exposición de las pinturas de Teresa Duclós recientemente adquiridas por la entidad que presido y con las que iniciamos un proyecto de colección de arte. [...] Una colección debe articularse mediante criterios claros. Pueden ser muy diferentes, por lo que se hace necesario optar y decidirse por aquéllos que puedan dar a la colección mayor interés y estén en mejor consonancia con el papel que debe cumplir una entidad como la nuestra. Hemos descartado algunos criterios, por ejemplo, aquél que busca abarcar muchos nombres cada uno escasamente representado. Nos parecía además que Caja San Fernando debía centrarse en el arte de calidad que se hace en Andalucía y en un período de tiempo relativamente abarcable. Ambos criterios señalan a los trabajos de los artistas andaluces de los últimos cuarenta años. Esta decisión no obedece a localismos ni tampoco a una idea de identidad diferencial andaluza, sino es más bien la respuesta a un fenómeno indudable: la personalidad y calidad de los trabajos de artistas andaluces a lo largo de estos años"10.

Las adquisiciones que responden a este proyecto de colección cuentan con un presupuesto de treinta millones de pesetas anuales y se prolongan solo durante tres años. En los mismos, la entidad adquiere la obra de seis artistas.

El bloque inaugural, en el año 2000, corresponde a la pintora sevillana Teresa Duclós, de la que se adquieren 26 óleos. El año 2001 es el más prolífico, ya que encuentran cabida en la colección autores como el malagueño Manuel Barbadillo (16 obras adquiridas), el sevillano Ignacio Tovar (18 obras) y el gaditano Chema Cobo (29 obras). En el año 2002, último en el que se adquiere obra, se compra un conjunto de 24 gouaches del grupo experimental afincado en Córdoba en la década de los cincuenta Equipo 57 y del pintor sevillano Joaquín Sáenz (29 obras).

El escaso recorrido del proyecto de colección se encuentra complementado, en cuanto a adquisiciones se refiere, con el Certamen de Artes Plásticas. La renovación del mismo, promovida por Francisco del Río, cuestiona el modelo tradicional de concurso en el que se presenta una única obra por artista. A partir de la edición XLI ${ }^{11}$, los participantes entregan un proyecto. El jurado lo forman

${ }^{9}$ La comisión se compone por el propio Paco del Río, el profesor de Estética de la Universidad de Sevilla Juan Bosco Díaz-Urmeneta, el profesor de Historia del Arte de la Universidad de Sevilla Fernando Martín Martín, el crítico de Arte Bernardo Palomo y el pintor José Soto.

${ }^{10}$ Díaz Urmeneta, 2001: 9.

11 Edición correspondiente al año 2002. 
artistas, profesores universitarios, críticos, comisarios y gestores de centros de arte, el cual seleccionará los mejores proyectos que los autores deberán realizar en el plazo de un año. Expuestos entonces, el jurado, reunido de nuevo, premiará uno de ellos y podrá recomendar la adquisición de otros ${ }^{12}$.

Fruto del remozado certamen llegarán a la colección de la Caja San Fernando obras de jóvenes autores que, bajo los criterios establecidos en el proyecto del año 2000, no habrían tenido cabida. De este modo, las creaciones de José Piñar, Simón Zabell, Juan Carlos Bracho, Julie Rivera o María José Gallardo recalan en los fondos.

En cuanto a El Monte, la elaboración de criterios profesionales se traza mediante un camino diferente. El proceso de consolidación de la colección abre una nueva etapa en el año 2000, fecha en la que su responsable, Francisco Pérez Valencia, presenta un plan de organización con el objetivo de marcar una única política de adquisición, con unos criterios de compra que toman como punto de partida las obras que ya posee la entidad: "A finales del año 2000, principios del 2001 es cuando presento a la entidad un plan de organización en el que comienzo a hablar de colección. Hasta el año 2000 yo empiezo primero orientando, siguiendo, cuidando, controlando y abriendo cada vez el camino de responsabilidad desde el año 94 hasta el año 2000 con mayor capacidad de decisión, ya no espero a que haya ofrecimientos, empiezo a tomar la iniciativa. La primera concepción es una especie de puzzle que empezamos reconstruyendo pensando en terminar el gran puzzle que sería la colección, entonces me doy cuenta que hay vacíos importantes que cubrir, ahí es cuando voy tratando de ofrecer obras para su adquisición. Así que, en el año 2000 comienzo a manifestar a don Juan Pedro Álvarez ${ }^{13}$ que debemos asumir un concepto de colección, a lo que él pregunta ¿Y qué colección se puede tener?"14.

En base a ese cuestionamiento se marcan unas líneas de actuación básicas para el crecimiento de la colección, dividiendo los fondos en compartimentos y estudiando qué obras son las necesarias para complementar lo existente.

Nace un espacio para el arte barroco andaluz. Aunque la colección no contaba con obra de este periodo histórico artístico, desde el punto de vista institucional se instó a iniciar una línea basada en el siglo XVII. El nacimiento de la casa de subastas Arte, Información y Gestión ${ }^{15}$, empresa participada por El Monte,

${ }^{12}$ El certamen tiene como última convocatoria la edición del año 2010, en la que son seleccionados los artistas François Bucher, Daniel Jacoby, Alessandra Sanguinetti y Jorge Satorre.

13 Juan Pedro Álvarez ostenta en ese momento el cargo de Director General de El Monte.

${ }^{14}$ Pérez Valencia, entrevista realizada el 11 de febrero de 2015 en Sanlúcar de Barrameda. Entrevistador: Juan María Vélez Alvez.

${ }^{15}$ Casa de subastas creada en el año 1998 y extinguida en el año 2013. 
resulta determinante en la compra de las piezas de esta centuria, ya que todas se adquieren en la misma. La institución decide apoyar de esta manera a la casa de subastas, así como rentabilizar mediáticamente las adquisiciones, debido a la amplia trascendencia y expectación con las que eran acogidas las citadas compras: "Nacen por recomendaciones institucionales otras líneas de trabajo como El Barroco andaluz, aparece en 1998 Arte, Información y Gestión y hay que apoyarla. Yo nunca hubiera comprado piezas tan caras como esas, prefería apuntalar autores jóvenes"16.

El siguiente capítulo de la colección es el correspondiente a la pintura andaluza del siglo XIX; en el mismo se recoge tanto la tendencia romanticista como la realista: "Del siglo XIX estaba todo más atado y se fueron cubriendo vacíos con compras puntuales. El siglo XIX viene un poco forzado, con sentido dentro de una institución como la nuestra, pero yo siempre pensé que era lo peor que había dado la Historia del Arte de nuestro país, mientras en el mundo había gente haciendo Manifiestos Futuristas, aquí se estaba pintando la estampa romántica de la bailaora y el bandolero. Sin embargo encontramos piezas pequeñas de paisajes adscritas a la Escuela de Alcalá y fuimos apuntalando y dando cohesión a piezas aisladas, estas dan rigor a lo que antes era un poco popurri'"17.

De manera paralela aparece una nueva expectativa hacia lo actual, donde los parámetros geográficos instalados en la raíz de la colección comienzan a abrirse hacia nuevas posibilidades más allá de las fronteras andaluzas. Se trata de una colección dentro de la colección, abierta a nuevos lenguajes, principalmente fotografía y vídeo y cuyo nombre es Escala Colección:

"Hay que apuntalar lo contemporáneo y tenemos que abrirnos hacia terrenos más allá del geográfico. Muchos artistas que siendo de aquí y trabajando aquí, su punto de mira no está aquí. Era la primera vez que estábamos entrando en territorios actuales.

En el 2000 con Escala Colección, una colección que comienza a crecer, permite ir llenando ese concepto que se termina convirtiendo en un concepto de colección global híbrida que es la que permite unos años después hablar de un plan museológico ${ }^{18}$.

La colección comienza a albergar la obra de artistas que crean en la actualidad, pasa de un criterio histórico a un criterio especulativo. Eso es Escala. A mí

${ }^{16}$ Pérez Valencia, entrevista realizada el 11 de febrero de 2015 en Sanlúcar de Barrameda. Entrevistador: Juan María Vélez Alvez.

${ }^{17}$ Pérez Valencia, entrevista realizada el 11 de febrero de 2015 en Sanlúcar de Barrameda. Entrevistador: Juan María Vélez Alvez.

${ }^{18}$ El único plan museológico desarrollado para la Colección Cajasol lo elabora Francisco Pérez Valencia en el año 2007. 
me apasionaba un concepto que había mencionado Paco Molina ${ }^{19}$, que el artista era sismógrafo de su momento histórico. Es el primer modelo de Colección del presente que después asume el MUSAC, nosotros asumimos eso con Escala Colección. Aspira a salirse de la matriz para mirar el presente sin importarle la posibilidad de errar, tú no puedes errar con un Gordillo, aunque tienes que tener presente su obra. De repente una colección que empieza a nacer verticalmente, empieza a tener raíces fractales, y salta, y se quiebra, y se rompe y no nos importa, eso le dio mucha frescura a la colección, mucha dinámica. Es una línea que se abre, que no aspira a llegar a ningún sitio, si con el tiempo alguno de estos autores se consolida en el terreno artístico entran a formar parte de la colección, si no, ha sido un terreno de especulación, sin miedo" ${ }^{20}$.

\section{EL MODELO DE COLECCIÓN DEFINITIVO: LA COLECCIÓN CAJASOL}

Como fruto de la fusión en el año 2007 de El Monte y Caja San Fernando nace Cajasol. Esta unión supone no solo la integración del negocio principal de ambas instituciones, el financiero, también se deben integrar equipos de trabajo y patrimonio. Dentro de este se sitúa la Colección de Arte.

En cuanto al ámbito de responsabilidades, los dos profesionales que dirigían las colecciones previas a la fusión se sitúan en el nuevo organigrama de la siguiente manera: Francisco del Río como responsable de exposiciones de la Obra Social y Cultural y Francisco Pérez Valencia como director de la Colección de Arte. Por tanto, corresponde a este último la gestión de los fondos de la entidad naciente.

La fórmula diseñada por Francisco Pérez Valencia opta por continuar, en buena medida, con los criterios establecidos en la Colección El Monte, elaborando una dirección de crecimiento orientada a cubrir un amplio espectro cronológico que nace desde el siglo XVII hasta el arte actual. Las líneas de adquisición potenciadas en la Colección Cajasol como consecuencia de los criterios de adquisición establecidos, cierran los grandes bloques en los que se dividen los fondos. Se trata de un total de cinco que a continuación se describen:

${ }^{19}$ Pintor y gestor de la actividad cultural de la Obra Social El Monte en los años ochenta. Fallece en 1993.

${ }^{20}$ Pérez Valencia, entrevista realizada el 11 de febrero de 2015 en Sanlúcar de Barrameda. Entrevistador: Juan María Vélez Alvez. 
1. Grandes maestros del Barroco andaluz.

Sin ánimo de tener presente en la colección una cantidad de obras que permitan realizar un recorrido exhaustivo por el Barroco andaluz, se centra en dar puntual testimonio de artistas concretos, componentes del primer eslabón de calidad de este periodo y con piezas representativas de su lenguaje plástico. Línea de trabajo de baja intensidad dentro de las adquisiciones, se compran cuatro obras en la primera década del siglo XX: Francisco de Zurbarán (San Pedro Nolasco asistido por dos ángeles), Bartolomé Esteban Murillo (Las dos Trinidades), Alonso Cano (Visión de San Antonio de Padua) y un anónimo (Vista de Triana desde el Arenal). Todas se adquieren en la casa de subastas Arte, Información y Gestión²1, pactándose puntualmente con la dirección general o presidencia de Cajasol, quienes por el fuerte desembolso que supone, se encargan de autorizar las mismas.

\section{Siglo XIX y principios del XX en Andalucía.}

La siguiente etapa representada es el siglo XIX. Al igual que la primera, se trata de una línea que concentra un bajo número de adquisiciones, las cuales, no obstante, vienen a completar unos fondos previos más amplios y representativos de la época.

Las adquisiciones efectuadas entre el año 2000 a 2010 fueron puntuales, cubriendo la representación de artistas relevantes que o no contaban con ninguna obra en los fondos o la obra con la que contaban era menor.

En el citado periodo se compran en este capítulo las siguientes obras: Gonzalo Bilbao (Concierto en el harem), José Pinelo (Paisaje de Alcalá de Guadaíra), José Arpa (Vista de la campiña) y Gustavo Bacarisas (Vista de Gibraltar). Todas estas piezas se adquieren en la casa de subastas Arte, Información y Gestión.

Estas obras vienen a complementar a los artistas presentes en la colección que trabajan a mediados del siglo XIX en las coordenadas del lenguaje romántico: Valeriano Bécquer, Antonio María Esquivel, Francisco de Paula Escribano; corrientes cercanas al realismo paisajista hacia finales del mismo siglo, representadas por José Jiménez Aranda, Emilio Sánchez Perrier o José Villegas; y, por último, a principios del siglo XX, estéticas próximas al folklore andaluz con Manuel García Rodríguez o José García Ramos. El 800 andaluz y, más concretamente el sevillano, constituye uno de los fuertes de la colección ${ }^{22}$.

${ }^{21}$ Las fechas de las adquisiciones son: Francisco de Zurbarán, 11 de noviembre de 1999; B. E. Murillo, 16 de noviembre de 2000; Alonso Cano, 14 de noviembre de 2002; y Anónimo, 7 de abril de 2000.

22 Bonet Planes, 2004: 21. 
3. Los inicios de la plástica moderna en Andalucía: años 60-70.

Con algunos antecedentes de carácter individual en la primera mitad del siglo XX, el camino hacia una nueva realidad plástica en el ámbito de la creación andaluza se inicia en la década de los 60. Esta línea de trabajo demandó mayores esfuerzos de investigación y documentación que las anteriores, ya que pretendía reconstruir un discurso exhaustivo de la época que aborda; no en vano es el inicio del hilo argumental de la Colección de Arte, centrada fundamentalmente en la creación contemporánea en Andalucía. Los fondos pertenecientes a esta línea son amplios y su contenido es muy completo. Las compras se dirigen a obras paradigmáticas de la estética de la época con el objetivo de complementar el discurso de los fondos existentes. El contenido se basa fundamentalmente en tres premisas:

1. Obra figurativa de artistas que con posterioridad se adentran en la abstracción: Miguel Pérez Aguilera, Jaime Burguillos, José Soto o Joaquín Meana.

2. Creadores que continuaron trabajando en un lenguaje figurativo: Carmen Laffón, Joaquín Sáenz, José Luis Mauri, Teresa Duclós, Santiago del Campo, Diego Ruiz Cortés, Juan Romero, Paco Cuadrado o Paco Cortijo.

3. Artistas que desarrollan su labor creativa en el ámbito de la abstracción: Manuel Barbadillo, José Ramón Sierra, Juan Suárez, Gerardo Delgado o Equipo 57.

Hay que añadir creadores que, tanto por el desarrollo de su trabajo como por su recorrido vital, aun coincidiendo cronológicamente con los anteriores, son de difícil clasificación, caso de Luis Gordillo y Nacho Criado.

Las obras de este periodo se adquieren principalmente a galerías aunque en alguna ocasión se realiza la transacción directamente con el artista mediante contrato de compraventa.

4. La consolidación de la modernidad: años 80-90.

Al igual que en el epígrafe anterior, los últimos veinte años del siglo XX cuentan con una perspectiva histórica necesariamente amplia como para poder abordar la construcción de un discurso complejo, completo y exhaustivo. A pesar de poder dividir estás últimas décadas en dos, ya que sobre todo, los años 80 contaron con una personalidad fuertemente marcada, considerada como la "década del entusiasmo" 23 , los artistas que desarrollan su trabajo en estos años constituyen una generación joven que continuará trabajando en la década posterior. Se

\footnotetext{
${ }^{23}$ Martín Martín, 1999: 51.
} 
trata, pues, de vasos comunicantes, fronteras difusas donde establecer divisiones cronológicas concretas es una tarea complicada.

La configuración de esta línea de trabajo dentro de la Colección de Arte implica una labor constante de documentación e investigación dirigidas a encontrar aquellas obras definitivas de los artistas destacados. Es difícil establecer categorías cerradas en la creación plástica de estos años, ya que prima el carácter individual de cada artista. En la abstracción se encuentran representados en los fondos pintores como José María Bermejo, Ignacio Tovar, Manuel Salinas o Pepe Barragán. Dentro de una figuración lírica se sitúan Rolando Campos, María Manrique, Carlos Montaño, Juan Lacomba o Félix de Cárdenas. Con un claro espíritu antirromántico en la concepción de la creación plástica se encuentra la joven generación surgida alrededor de la revista Figura: Federico Guzmán, Patricio Cabrera, Guillermo Paneque, Pepe Espaliú, Rafael Agredano, Ricardo Cadenas y Curro González. Compañeros generacionales cercanos a estos planteamientos son Antonio Sosa y Gonzalo Puch. Algunos de los artistas andaluces vinculados a la Nueva Figuración Madrileña de los años setenta están presentes en la colección con obra en su mayoría perteneciente a los ochenta. Son los casos de Guillermo Pérez Villalta, Chema Cobo, Alfonso Fraile o Manolo Quejido.

Otros artistas que forman parte de la colección, cuyo trabajo está vinculado a estas décadas, siendo su labor de difícil clasificación puesto que su trayectoria es marcadamente personal, son Paco Molina, Fernando Zóbel, Paco Reina, Pedro Simón, José María Báez o Rafael Zapatero.

Una línea intermedia de adquisiciones es la que da cabida a un grupo de artistas nacidos en los años sesenta. Estos comienzan a obtener el reconocimiento a su trabajo en la segunda mitad de los noventa. Se trata de una generación intermedia entre los ochenta y principios de los noventa y la joven generación actual -la cual tiene un epígrafe individual en las adquisiciones-. De este grupo de artistas se encuentran representados en los fondos Javier Velasco, Alonso Gil, José Piñar, Mercedes Carbonell, Chema Alvargonzález, Juan Carlos Robles, Dionisio González, Pepa Rubio, Ángeles Agrela o Carmen Sigler.

\section{La actualidad: siglo XXI.}

La creación actual es la que ha requerido un uso de recursos más elevado en la Colección de Arte, tanto a nivel de inversiones como de ocupación de los recursos humanos. Esta línea de trabajo se orienta principalmente a dos objetivos: el apoyo a jóvenes creadores andaluces y la búsqueda de autores fuera del ámbito andaluz ${ }^{24}$.

${ }^{24}$ Esta línea de trabajo es la denominada por Francisco Pérez Valencia como Escala Colección. 
El primero de estos objetivos pretende dar cabida a la pujante generación de artistas plásticos de la comunidad andaluza. Un numeroso grupo de creadores muy activos nacidos en los años setenta y principio de los ochenta. Comprometidos con lo contemporáneo, han contado con unas posibilidades formativas y de acceso a canales artísticos superior a las generaciones precedentes. Probablemente es el grupo cuantitativa y cualitativamente más prolífico del panorama nacional en la actualidad.

El seguimiento de estos creadores en el mercado se divide en una triple línea de trabajo orientada a incluir a los creadores no presentes en la colección, contextualizar la obra de los artistas ya representados con sus nuevas creaciones y la producción de proyectos para exposiciones de la propia colección.

En este apartado, los artistas que cuentan con obra en los fondos son Carlos Aires, Manolo Bautista, Juan Carlos Bracho, María Cañas, María José Gallardo, Noelia García Bandera, David López Panea, Miki Leal, Gloria Martín, José Miguel Pereñíguez, MP\&MP Rosado Garcés, Julia Rivera, Jorge Yeregui, Simón Zabell y Jesús Zurita.

El segundo objetivo busca creadores en otros ámbitos geográficos, haciéndose eco de un mundo cada vez más globalizado. En cierto modo se acompaña la inercia vital de Cajasol a comienzos del siglo XXI, en la que la expansión hacia parte del territorio nacional era una realidad y, a nivel creativo, las inquietudes artísticas se generalizan creando discursos paralelos con independencia del lugar de origen del creador, se comenzó una nueva línea de adquisiciones abiertas desde el punto de vista geográfico.

El criterio de adquisición es mantener un firme compromiso con lo contemporáneo a nivel conceptual y estético, además las piezas adquiridas deben ser representativas en la producción del autor. Esta línea obligó a establecer un seguimiento constante de la programación de centros de arte contemporáneo, galerías y la asistencia a las ferias más importantes a nivel nacional (ARCO, LOOP y la extinta DFOTO en San Sebastián). Según un criterio geográfico, las adquisiciones de dividen en tres territorios:

1. Creación española. Se combinan obras de creadores referentes en la plástica nacional, cuya trayectoria está ampliamente contrastada, como Alberto García Álix, Cristina García Rodero, Joan Fontcuberta, José Manuel Ballester, Chema Madoz o Bleda\&Rosa, con artistas cuyo trabajo comienza a despuntar. Es el caso de Maider López, Raúl Belinchón, Nico Munuera, Mayte Vieta o Eugenio Ampudia.

2. Creación sudamericana. En la misma línea de compromiso con discursos actuales se han adquirido obras de Óscar Muñoz, Diango Hernández, Deborah Castillo, Sebastián Díaz Morales, Federico Herrero, Juan Pablo Ballester o Maykel Linares.

3. Creación europea y norteamericana. Las adquisiciones en esta línea fueron muy numerosas, primando el ámbito europeo aunque con algún 
complemento norteamericano. Algunos de los autores representados son Eva Koch, Robert Pollidori, Edward Burtynsky, Phil Collins, Patrick Jolley \& Reynold Reynolds, Risk Hazekamp, Larry Sultan, Johanna Domke, Mariana Vassileva, Paulo Nozolino, Jorge Molder, Pierre Gonnord, Rut Blees Luxemburg o Thomas Weimberger.

Las líneas de adquisición diseñadas por Francisco Pérez Valencia para El Monte, continuadas y potenciadas tras la fusión en la que verá la luz una nueva caja de ahorros, Cajasol, se acompaña de un esfuerzo inversor que alcanza su punto álgido en el año 2007 con un presupuesto dedicado a la compra de obra que alcanza la cifra de $220.000 €^{25}$. Las nuevas incorporaciones a la colección provienen de un sondeo arduo del mercado del arte. Las galerías de arte contemporáneo son las principales proveedoras, ya que en las obras creadas de aquel momento y los nuevos medios digitales ocupan el mayor porcentaje de piezas adquiridas.

El presupuesto para las inversiones en obras de arte mengua considerablemente en el año 2008 como consecuencia de la reducción presupuestaria derivada de la crisis financiera. La marcha de Francisco Pérez Valencia de la entidad en 2010 y el fallecimiento de Francisco del Río en 2011, suponen el ocaso de un ciclo en la Colección de Arte Cajasol. El final de un proyecto vinculado a la creación de un equipo de trabajo profesional de perfil técnico, que no se ha vuelto a recomponer con nuevos nombres ni iniciativas para el crecimiento de la colección.

A partir de 2011 la institución se verá abocada a un proceso de cambios institucionales que deriva en la actual Fundación Cajasol de carácter privado. Desde el año 2009 al año 2019 solo se contabilizan cinco compras de entidad para su colección ${ }^{26}$.

La suma de fondos atesorados en casi 175 años de historia y la gestión profesional de menos de dos décadas (1993-2011) tienen como resultado la más notable colección de arte en manos privadas de Andalucía. Según el inventario finalizado en 2014, cuenta en la actualidad con 4.351 obras. Los fondos son el resultado de diferentes etapas cuyo logro principal ha sido constituir un conjunto singular en el panorama del coleccionismo institucional en Andalucía.

La Colección Cajasol se muestra capaz de representar un recorrido amplío, a la vez que sólido, por diferentes épocas del arte andaluz, implementando varias líneas de actuación cronológica, con capítulos dedicados al arte del siglo XVII y

${ }^{25}$ Pérez Valencia, entrevista realizada el 11 de febrero de 2015 en Sanlúcar de Barrameda. Entrevistador: Juan María Vélez Alvez.

${ }^{26}$ Las obras adquiridas en ese periodo son: Obrador de Murillo, San José con el niño (2009); Valdés Leal, Cristo atado a la columna (2012); Pedro de Campaña, Calvario (2013); Andrés Cortés y Aguilar, Vistas de las afueras de Sevilla (2013); y Luis Gordillo, Cabeza Bacon (2019). 
XIX, además de resultar la colección que con mayor profundidad recoge la diversa producción andaluza de la segunda mitad del siglo XX. Esto se complementa con una visión de lo contemporáneo amplia y versátil, ocupándose tanto del arte joven, como de representar la imagen fotográfica y digital en el ámbito nacional e internacional del último cambio de siglo.

Fecha de recepción: 16 de octubre de 2019

Fecha de aceptación: 13 de abril de 2020

\section{BIBLIOGRAFÍA}

Bonet Planes, Juan Manuel (2004): "Variaciones en torno a la Colección El Monte". En: Bonet, Juan Manuel/ Pérez, Francisco (coms.): El Arte habitado. Sevilla: Fundación El Monte, pp. 21-39.

Campayo Rodríguez, Cristina (1992): El Monte y Sevilla. Un siglo y medio de Historia. Sevilla: Fundación El Monte.

Díaz Urmeneta, Juan Bosco (2001): Teresa Duclós. Sevilla: Caja San Fernando.

Díaz Urmeneta, Juan Bosco/Marina, Alberto/Ramos, Rosario (2012): Paco del Río. Del arte y los afectos. Sevilla: Fundación Cajasol.

Martín Martín, Fernando (1999): "Crónica de un vacío: el coleccionismo de arte contemporáneo en Sevilla”. En: Chacón, José Antonio/Fernández, Josél Martín, Fernando/Rodríguez, Francisco Javier (coms.): La colección de El Monte. Sevilla: Fundación El Monte, pp. 39-76.

(2010): "Crisis, mercado y coleccionismo en Andalucía”. En: Laboratorio de Arte, 22, pp. 479-489.

Palomo Pachón, Bernardo (2004): La renovación plástica en Andalucía. Desde el Equipo 57 al CAC Málaga. Málaga: CAC Málaga/Gestión Cultural y Comunicación.

Pérez Valencia, Francisco (1994): Colección El Monte, Caja de Huelva y Sevilla. Sevilla: Fundación El Monte. 